\title{
Efficient parallel timing simulation of synchronous models on networks of workstations
}

\author{
Edwin Naroska ${ }^{a, *}$, Feipei Lai ${ }^{b}$, Rung-Ji Shang ${ }^{c}$, Uwe Schwiegelshohn ${ }^{a}$ \\ a Computer Engineering Institute, University of Dortmund, Dortmund, Germany \\ ${ }^{\mathrm{b}}$ Department of Computer Science and Information Engineering and Department of Electrical Engineering, National Taiwan University, \\ Taiwan, $R O C$ \\ ${ }^{\mathrm{c}}$ Department of Computer Science and Information Engineering, National Taiwan University, Taiwan, ROC
}

\begin{abstract}
In this paper we address the parallel timing simulation of synchronous VLSI designs on a network of workstations (NOWs). We suggest combining cycle based and conventional timing simulation techniques to achieve fast timing simulation even on NOWs which are typically characterized by low bandwidth and high communication latency. In particular we execute a timing simulator on each node of the NOW and use cycle based simulation to produce synchronization information required by the timing simulators. As synchronization information is generated exclusively by the cycle based simulator there is no need for any communication between the timing simulators. To verify the feasibility and performance of our approach we simulated several circuits using our approach. The results show that a significant speedup can be achieved even for very small circuits. (c) 2001 Elsevier Science B.V. All rights reserved.
\end{abstract}

Keywords: Parallel timing simulation; Cycle based simulation; Network of workstations

\section{Introduction}

This paper addresses the use of combined cycle based and timing simulation to speedup timing verification of VLSI designs. Cycle simulators achieve their performance gain over timing simulators by determining the state of synchronous circuitry at clock boundaries only. As cycle simulators provide only minor timing information they are backed up by static timing analysis to check timing behavior.

\footnotetext{
${ }^{*}$ Corresponding author.

E-mail addresses: edwin@ds.e-technik.uni-dortmund.de (E. Naroska), flai@cc.ee.ntu.edu.tw (F. Lai), shang@bulls.csie. ntu.edu.tw (R.-J. Shang), uwe@ds.e-technik.uni-dortmund.de (U. Schwiegelshohn).
}

Static timing analyzer detect timing violations by investigating the gate and wire delays of a design. In particular, the analyzer sums up all gate and wire delays for all paths within a circuit. If it turns out that the delay along a specific path is longer than the clock cycle time then this path is reported to the designer. However, besides real timing violations these tools tend to report false paths which must be checked by the designer manually. False paths have a path delay longer than the clock cycle time but are never activated during normal operation of the circuit, i.e., during real operation there will be no transition propagating from the beginning to the end of the path. The manual checking of large and complex designs is usually unacceptable.

Detailed timing simulation on the other hand does not require assistance by the designer. 
Further, timing simulation is used for accurate power calculation which is attracting more and more attention these days. However, due to the simulation workload large designs cannot be handled efficiently by common simulation platforms.

Therefore, timing simulation on a parallel computer or a network of workstations (NOWs) has been suggested to speed up circuit verification. This approach is based on the parallel discrete event simulation (PDES) paradigm [1-5, 7-10,14].

Previous research in PDES has resulted in two main simulation approaches, the conservative and the optimistic one [1-3,7]. In the conservative approach all events associated with a specific process (i.e., gate or flipflop) are executed in time-stamp order $[10,11]$. To preserve correctness of the simulation the processing nodes periodically generate and exchange information about the current state of the simulation with each other.

Alternatively, the optimistic approach allows execution of an event at a process (i.e., gate or flipflop) without guaranteeing that no other event with a smaller time stamp may later arrive at the same process $[8,13]$. If the chronological order is violated, the simulation must be rolled back in time to a safe state. Therefore, an optimistic simulation algorithm requires a strategy for periodically backing up the simulation. This results in additional memory requirements and may be unacceptable for large VLSI simulation tasks.

In conservative as well as optimistic approaches the processing nodes communicate with each other in order to synchronize themselves $[1-3,5,8,10]$. As communication is slow compared to computation this mechanism often becomes a bottleneck during simulation. In particular, NOWs are characterized by a high latency and low bandwidth communication layers. Hence, in spite of their excellent availability and good price performance ratio NOWs are not well suited for common PDES approaches. Using a dedicated high speed communication network partly widens the communication bottleneck but it comes at a high cost. But even when exploiting high speed networks the speedup is often limited $[4,10]$. Alternatively, parallel simulation can be run on shared memory system [5]. While these systems provide efficient communication resources affordable systems usually include only a small number of processors.

Another important issue for PDES is partitioning. In common parallel simulation the model is partitioned and each part is assigned a different computation node. Hence, each node is executing another part of the model for the same virtual simulation time span. While partitioning reduces the memory consumption on each node a good partitioning is often hard to find. During partitioning the memory consumption, the computation workload as well as the communication workload must be balanced among all nodes. This is hard to achieve as computation and communication workloads are usually data dependent.

In contrast to the common PDES techniques, our approach does not need any partitioning nor does it require any communication between the computation nodes during simulation. However, each simulation node must be able to hold the entire model in memory.

\subsection{Discrete event and cycle based simulation algorithms}

Before our approach is explained in detail an overview on discrete event as well as cycle based simulation algorithms is given using the model shown in Fig. 1.

The model has two primary inputs $i_{0}$ and $i_{1}$ and two outputs $q_{0}$ and $q_{1}$. It consists of four flipflops $f f 1-f f 4$ and three gates $g 1-g 3$. The internal signal nets are labeled $a, b, c$ and $d$. Finally, the flipflops are associated with a propagation delay of $4 \mathrm{~ns}$ while the gates have a delay of $2 \mathrm{~ns}(g 1), 3 \mathrm{~ns}(g 2)$ and $1 \mathrm{~ns}(\mathrm{~g} 3)$.

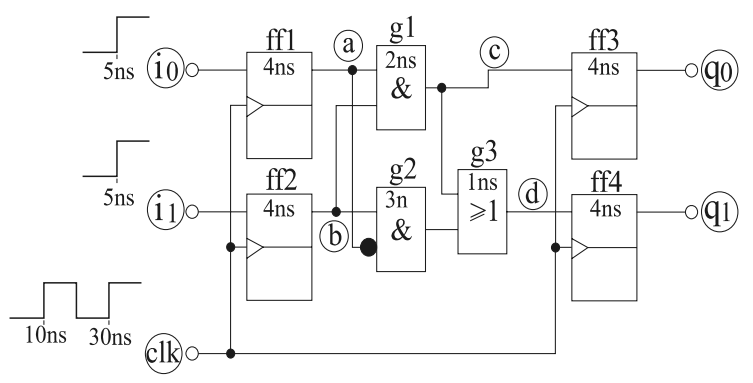

Fig. 1. A simple digital model. 
The basic data structure of a discrete event simulation is the event queue which stores all events of the model in increasing time stamp order. An event consists of a value and a time stamp. The time stamps determines the virtual time at which the value shall be assigned to the corresponding signal.

The discrete event simulation cycle consists of three steps:

1. The current simulation time is set to the time stamp of the event stored in the head of the event queue.

2. The values of all events with a time stamp equal to the current simulation time are assigned to the corresponding signals. Thereafter, the events are removed from the system.

3. All flipflops and gates connected to a signal which has changed its value in step 2 are executed. Due to the execution new events may be created and inserted into the event queue.

Each row in Table 1 presents the content of the global event queue for the model shown in Fig. 1 at the end of the corresponding simulation cycle. The notation of an event is (val,t)@sig, where val is the value that will be assigned at simulation time $t$ to signal sig. To stimulate circuit activity signals $i_{0}, i_{1}$ and $c l k$ are assigned events as shown in Fig. 1. All signal values are assumed to be 0 at simulation start. For the sake of brevity only the next pending event of signal $c l k$ is listed in Table 1.

The first row of the table shows the content of the event queue at simulation startup. As the next earliest time stamp on the queue is 5 ns the next simulation time is set to $5 \mathrm{~ns}$ and the two first events are assigned to signals $i_{0}$ and $i_{1}$. This causes

\section{Table 1}

Content of the global event queue during discrete event simulation

\begin{tabular}{llll}
\hline Time (ns) & Head & $\rightarrow$ & Tail \\
\hline 0 & $(1,5 \mathrm{~ns}) @ i_{0}$ & $(1,5 \mathrm{~ns}) @ i_{1}$ & $(1,10 \mathrm{~ns}) @ c l k$ \\
5 & $(1,10 \mathrm{~ns}) @ c l k$ & & \\
10 & $(1,14 \mathrm{~ns}) @ a$ & $(1,14 \mathrm{~ns}) @ b$ & $(0,20 \mathrm{~ns}) @ c l k$ \\
14 & $(1,16 \mathrm{~ns}) @ c$ & $(0,20 \mathrm{~ns}) @ c l k$ & \\
16 & $(1,17 \mathrm{~ns}) @ d$ & $(0,20 \mathrm{~ns}) @ c l k$ & \\
17 & $(0,20 \mathrm{~ns}) @ c l k$ & & \\
20 & $(1,30 \mathrm{~ns}) @ c l k$ & & \\
30 & $(0,34 \mathrm{~ns}) @ q_{0}$ & $(1,34 \mathrm{~ns}) @ q_{1}$ & \\
34 & & & \\
\hline
\end{tabular}

flipflops $f f 1$ and $f f 2$ to be evaluated. However, as the flipflops are activated by a rising edge on their clock inputs no output events are generated. Hence, the next simulation time is $10 \mathrm{~ns}$. Now, all flipflops are activated. However, only $f f 1$ and $f f 2$ create an event with time stamp 14 ns for their output signals $a$ and $b$. Flipflops $f f 3$ and $f f 4$ actually do not schedule a value change on their outputs. Hence, no events are generated by $f f 3$ and $f f 4$.

Obviously, a significant part of the simulation runtime is spent maintaining the global event queue during simulation (i.e., inserting and removing events from the queue). Besides the event handling overhead additional runtime is spend to evaluate flipflops and gates whenever their input signals make a transition.

The event handling overhead is avoided by the cycle simulation technique as no event queue is used to keep track of the activity within the circuit. Instead, all signals values are recalculated in each simulation cycle.

Fig. 2 shows the cycle based simulation program that was generated from the model shown in Fig. 1. Note that the program sequence to set up the values for the input signals $i_{0}$ and $i_{1}$ are not shown in the Figure. Each time the procedure simulation_cycle is called the signal values right after the next rising clock edge are determined and immediately assigned to the signals without any delay. Hence, no timing information can be derived from the cycle simulation, i.e., the simulation programs operates as if all gate and flipflop delays were zero.

Calling the procedure twice will actually simulate the same virtual time span as shown by Table 1. Obviously, taking the event handling

$$
\begin{aligned}
& \text { procedure simulation_cycle is } \\
& \text { begin } \\
& \qquad \begin{array}{l}
q_{0}=(a \text { AND } b) \\
q_{1}=(a \text { AND } b) \text { OR }(b \text { AND NOT } a) ; \\
\quad \begin{array}{l}
a=i_{0} ; \\
b=i_{1} ;
\end{array} \\
\text { end procedure simulation_cycle; }
\end{array}
\end{aligned}
$$

Fig. 2. Cycle based simulation program generated from the sample model. 
overhead into account, the cycle simulation program will execute significantly faster than the corresponding discrete event simulator.

\section{Hybrid simulation approach}

In order to exploit the computation power of NOWs for parallel simulation, synchronization of the computation nodes must be reduced to a minimal amount. To achieve this goal we use cycle based simulation to generate synchronization information. Because cycle based simulation usually executes one to two orders of magnitude faster than timing simulation the synchronization information can be generated very fast [12]. This so called state information is then loaded into the timing (discrete event) simulators to start them at certain time instances. This technique limits communication to a minimal amount or shifts it into less critical regions. Further, as the cycle based simulators as well as the timing simulators used in our approach are based on well known serial simulation algorithms any enhanced techniques for serial simulation, as, e.g., proposed in [6], can be incorporated into our approach as well.

As cycle simulators provide only minor timing information we must first identify the elements of the circuit which shall be recorded during cycle simulation as well as the virtual points in time where the values of these elements match those of a corresponding timing model. Analyzing the operation of synchronous circuitry shows that the signal values of flipflop data inputs are equal in both the cycle and the timing simulator right before the active edge of the clock signal. However, this is only true if these signals reach a stable state before the next active clock edge. If this precondition is violated then the state information calculated by the cycle simulator differs from the corresponding signal values generated by a conventional timing simulation. But this situation represents a timing violation and must be reported to the designer anyway. Moreover, continuing timing simulation based on this wrong state transitions might not produce any useful information.

The main idea behind our approach is to use a combination of cycle based and timing simulation to reduce or remove the need of communication during parallel simulation. Our "hybrid" simulation technique makes use of cycle based simulation to produce synchronization information in order to run timing simulators in parallel. Each simulation type (cycle and timing) now in turn can be performed in two different ways. The simulators may either simulate the same model but for different (virtual) time slices (time approach) or they may simulate a different part of the model for the same (virtual) time slice (space approach). Following this convention a parallel simulation can be characterized by $\operatorname{Sim}($ Cycle $=X, \operatorname{Con}=Y)$ with $X, Y \in\{$ Time, Space $\}$, where Cycle determines the method used for cycle based simulation and Con is the method used for timing simulation.

By permuting the values of Cycle and Con we get four different simulation methods.

- $\operatorname{Sim}($ Cycle $=$ Time, Con $=$ Time $) . \quad$ Each $\quad$ cycle based simulator executes the whole model for a different (virtual) simulation time span. Note, all cycle simulators start at virtual time 0 but simulate up to a different virtual end time (see left column of Fig. 3(a)).

Each timing simulator is assigned a different virtual time slice. However, all timing simulators execute the entire model (see right column of Fig. 3(a)).

- $\operatorname{Sim}($ Cycle $=$ Space, Con $=$ Time $)$. Each cycle based simulator is assigned a different part of the model to perform a parallel cycle based simulation, i.e., all cycle simulators cooperate to simulate the entire model from 0 to $t_{\text {sim }}$ (see left column of Fig. 3(b)).

Each timing simulator on the other hand simulates the whole circuit but for a different virtual time slice (see right column of Fig. 3(b)).

- $\operatorname{Sim}($ Cycle $=$ Time, Con = Space $)$. This method is different compared to the other approaches described here because only a single cycle based simulator is used to produce state information. Further, each node is executing a different part of the model for the same virtual simulation time during timing simulation (see Fig. 3(c)).

Actually, this approach is very similar to conventional parallel simulation algorithms where 

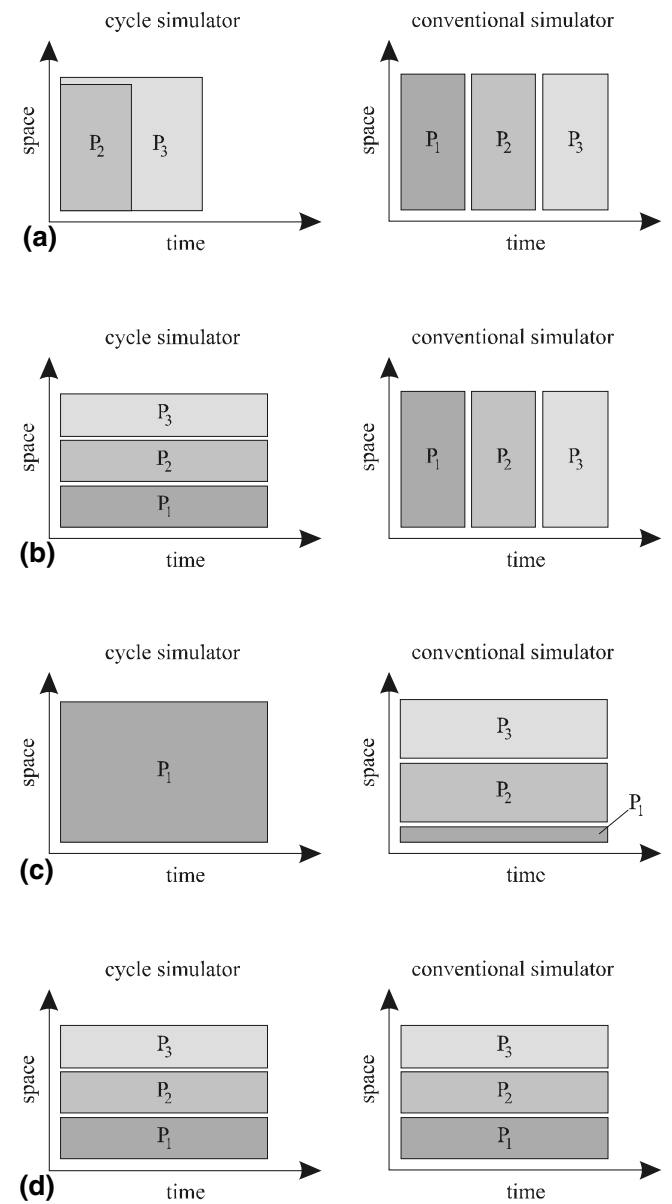

Fig. 3. Execution schema of the cycle and timing simulators. The time axis represents the virtual time and the space axis the elements of the model.

synchronization information is exchanged between the nodes to preserve correctness of the simulation. However, in this approach the information is exclusively produced by a single cycle simulator. Because the cycle simulator executes significant faster than the timing simulators, synchronization information will be created and distributed before it is needed by the timing simulators.

- $\operatorname{Sim}($ Cycle = Space, Con = Space $)$. Each cycle based simulator is assigned a different part of the model. Similar to algorithm Sim $($ Cycle = Space, Con $=$ Time) a parallel cycle simulation is used to generate state information (see left column of Fig. 3(d)).
Each timing simulator executes a different part of the model but for the entire virtual time span 0 to $t_{\text {sim }}$ (see right column of Fig. 3(d)).

The major advantage of the first approach $\operatorname{Sim}($ Cycle $=$ Time, Con $=$ Time $)$ is that no communication between the different computation nodes is required during simulation. Hence, this approach is very well suited for parallel simulation on NOWs. Therefore, we focus on this approach in the remaining parts of the paper.

In the following sections the simulation method as well as theoretical and experimental performance results are discussed. For the simulation task we assume a synchronous circuit at gate level with a clock cycle time of $t_{\text {clock}}$. The flipflops of the design sample their input signals at the active (rising or falling edge) clock edge. We assume that there is no clock delay along the clock path, hence all flipflops are activated simultaneously. The model is simulated on a parallel computer or NOW with $N$ computation nodes $P_{1}, \ldots, P_{N}$ from virtual time 0 to $t_{\mathrm{sim}}$. We assume that the real time to execute both a cycle and a timing simulator is proportional to the virtual simulated time span. Finally, we define $S$ to be the ratio between the real simulation time of a timing simulator compared to that of a cycle based simulator executing the same model for the same virtual time span. Note that $S$ is model and data dependent

$$
S=\frac{\text { timing simulation runtime }}{\text { cycle based simulation runtime }} \text {. }
$$

\section{1. $\operatorname{Sim}($ Cycle $=$ Time, Con $=$ Time $)$}

The simulation approach is restricted to synchronous circuits which do not include any logic cycles, i.e., each cyclic path must cross at least one flipflop.

We divide the simulation interval $\left[0, t_{\text {sim }}\right]$ for timing simulation into $N$ time slices. The main approach is to assign each time slice to a different timing simulator instead of simulating the entire interval on a single simulation engine. Hence, except the simulator, which is assigned the interval starting from 0 , all timing simulators will start from a different simulation time greater than 0 . As 
the state of the flipflops is unknown in advance, cycle simulation is used to determine their values for each timing simulation starting point. These values are then preloaded into the corresponding timing simulators to start timing simulation.

However, cycle simulation generates only static values for all signals in a clock cycle, i.e., all transitions in a cycle simulation take place at clock boundaries. Hence, no detailed dynamic timing information about time instances signal transitions take place can be derived from a cycle simulation run. Moreover, in the timing model some signal values within the circuit may still float following an active clock edge without violating any setup and hold timing condition of the flipflops while in the cycle model these signals are stable right after the active clock edge.

When the simulation state is transferred from the cycle to the timing simulator then all signals in the timing simulator are assigned a start value. No information about the time instances when these transitions actually took place is available. Hence, a technique is needed to determine the correct dynamic waveforms for all signals within the model based on the state information provided by the cycle simulator.

This can be achieved by starting timing simulation "some" cycles earlier. After this startup phase the timing behavior of all signals will exactly match those of the circuit obtained with a serial timing simulator with start time 0 . The length of the startup phase $t_{\mathrm{su}}$ depends on the properties of the circuit.

Let $t_{\mathrm{sp}, \text { comb }}$ be the longest static delay for all paths of the combinational logic starting at any gate or flipflop input of the circuit and ending either at a flipflop input or at a primary output of the circuit. Then $t_{\mathrm{sp}, \mathrm{comp}}$ is rounded up to the next clock cycle boundary and an additional clock cycle is added to cover any delays of input signal fed into the circuit. With $\lceil x\rceil$ denoting the smallest integer greater or equal to $x, t_{\mathrm{su}}$ is

$t_{\mathrm{su}}=t_{\text {clock }} \times\left(1+\left\lceil\frac{t_{\mathrm{sp}, \mathrm{comp}}}{t_{\text {clock }}}\right\rceil\right)$.

Prior to simulation the following steps are necessary:
1. Two simulators are generated from the circuitry: a cycle based simulator for state precalculation and a conventional discrete event simulator for timing simulation.

2. The whole simulation time interval is divided into $N$ slices $t_{i}, i=1, \ldots, N$, with $\sum_{i} t_{i}=t_{\text {sim }}$.

Then, parallel simulation is executed as follows:

1. On node $P_{1}$ a timing simulator is immediately started to proceed to simulation time $t_{\mathrm{con}, 1}=t_{1}$.

2. On each remaining node $P_{i}, 2 \leqslant i \leqslant N$ a cycle simulator is started to proceed to time $t_{\mathrm{cyc}, i}=-t_{\mathrm{su}}+\sum_{j=1}^{i-1} t_{j}$.

The state of the cycle simulator is saved as state $_{i, 1}$ at $P_{i}$. Cycle simulation is continued to time $t_{\mathrm{cyc}, i}+t_{\mathrm{su}}$ saving the state of the model at each clock cycle. This provides a set of states state $_{i, 1}$ to state $_{i, k}$ where $k=t_{\mathrm{su}} / t_{\mathrm{clock}}$. After reaching $t_{\mathrm{cyc}, i}+t_{\mathrm{su}}$ the cycle simulator is terminated.

Next, state $_{i, 1}$ is preloaded into the timing simulator on node $P_{i}$ and the detailed timing model is simulated from $t_{\mathrm{cyc}, i}$ to $t_{\mathrm{cyc}, i}+t_{\mathrm{clock}}$. Then state $_{i, 2}$ is loaded into the model and simulation is continued to $t_{\text {cyc }, i}+2 \times t_{\text {clock }}$. This procedure continues until the last state information state $_{i, k}$ has been used. Any timing violations occurring during the startup interval $\left[t_{\mathrm{cyc}, i}, t_{\mathrm{cyc}, i}+t_{\mathrm{su}}\right]$ are ignored. Finally, timing simulation is continued to $t_{\mathrm{con}, i}=\sum_{j=1}^{i} t_{j}$.

Fig. 4 shows an example simulation running on three processors. Note that Processor 1 begins timing simulation at time 0 . Hence, it does not need to run a cycle simulator.

It is possible to select the values for $t_{i}$ such that all timing simulators will complete their execution simultaneously thus providing optimal speedup. In order to achieve this goal the values $t_{i}$ must match

$$
\begin{aligned}
t_{1} & =\frac{t_{1}}{S}+t_{2}+t_{\mathrm{su}}=\frac{t_{1}+t_{2}}{S}+t_{3}+t_{\mathrm{su}}=\cdots \\
& =\frac{\sum_{j=1}^{N-1} t_{j}}{S}+t_{N}+t_{\mathrm{su}}=\frac{t_{\mathrm{sim}}}{A_{T T}},
\end{aligned}
$$

where $A_{T T}$ denotes the speedup of the parallel simulation. In order to match Eq. (2) $S$ is assumed to be constant over the simulation interval $\left[0, t_{\mathrm{sim}}\right]$. Whether this predication is valid depends on the model and the input stimuli presented to the circuit. 


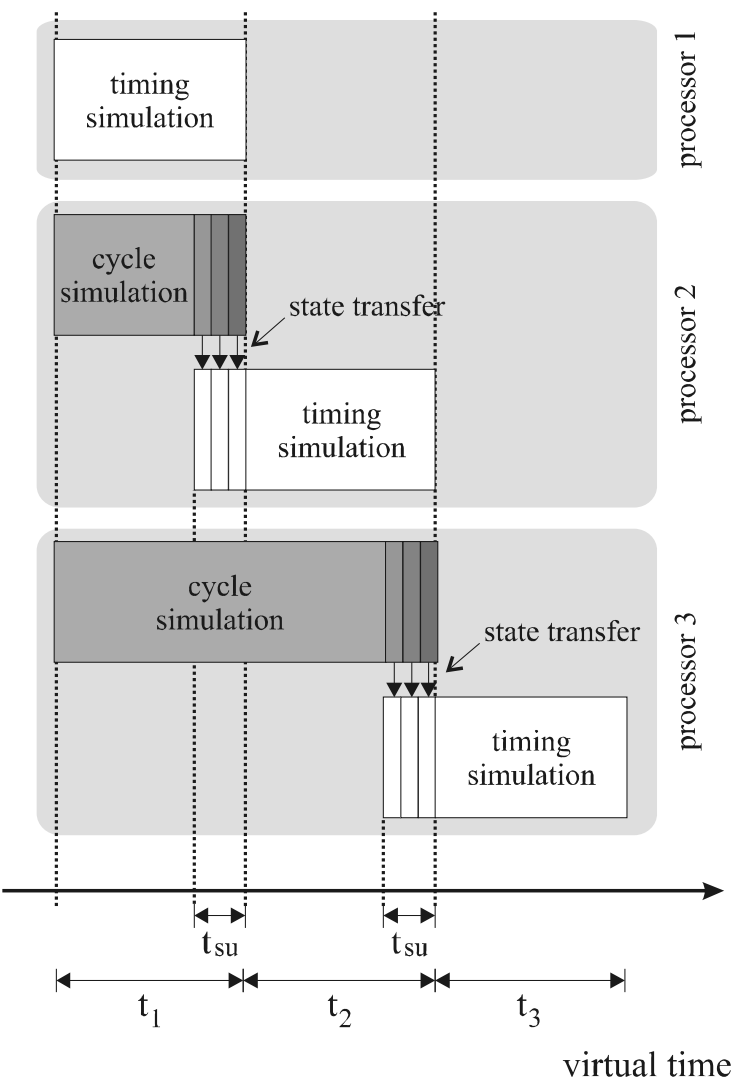

Fig. 4. Parallel simulation using three nodes.

Using $\sum_{i=1}^{N} t_{i}=t_{\text {sim }}$ with Eq. (2) yields the optimal value for $t_{1}$

$t_{1}=t_{\mathrm{sim}} \times \frac{\Omega-1}{\Omega^{N}-1}+t_{\mathrm{su}} \times \frac{\Omega^{N-1}-1}{\Omega^{N}-1}$,

where $\Omega=(S-1) / S$. The remaining time intervals $t_{i}(i \geqslant 2)$ are

$t_{i}=\left(t_{1}-\frac{t_{\mathrm{su}}}{\Omega}\right) \times \Omega^{i-1}$.

The optimal speedup is

$$
\begin{aligned}
A_{T T} & =\frac{\Omega^{N}-1}{\Omega-1+\left(t_{\mathrm{su}} / t_{\mathrm{sim}}\right) \times\left(\Omega^{N-1}-1\right)} \\
& =\frac{\Omega^{N}-1}{\Omega-1} \leqslant S \quad \text { for } t_{\mathrm{su}} \ll t_{\mathrm{sim}} .
\end{aligned}
$$

Eq. (5) is an upper bound for the speedup as no overhead for storing and loading the states is in-

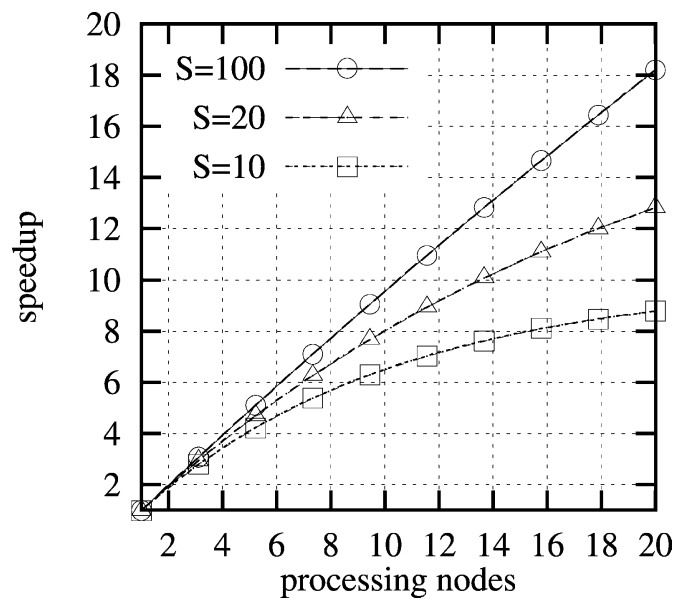

Fig. 5. Speedup for algorithm $\operatorname{Sim}($ Cycle $=$ Time, Con $=$ Time $)$.

cluded. Fig. 5 shows the theoretical speedup for $S=10, S=20, S=100$ with $t_{\mathrm{su}} \ll t_{\mathrm{sim}}$.

A significant advantage of this method is that no communication is required between the nodes during simulation. Hence, this method is well suited for parallel simulation on NOWs. However, each simulation node must provide sufficient memory to hold the whole model in memory. Further, this method is restricted to synchronous acyclic circuits.

\section{Experimental results}

In order to reduce the cycle time of the design flow we implemented a modified version of $\operatorname{Sim}($ Cycle $=$ Time, Con $=$ Time $)$. We developed a cycle based VHDL simulator which is capable of recording the state of the circuit as well as the input stimuli presented to the circuit during simulation. Hence, during cycle based simulation the function of a design can be verified while simultaneously recording state and input signals for a subsequent parallel simulation. Further we extended our serial VHDL simulator to load state information and feed prerecorded input stimuli into the model. Storing/accessing state information and input stimuli is implemented via file read/write operations. 
In the modified approach the cycle simulator simulates the model up to the same virtual time as the corresponding timing simulator in order to create a complete set of input stimuli. Hence, to obtain an optimal speedup $A_{T T, \text { mod }}$ the values $t_{i}$ must satisfy

$$
\begin{aligned}
t_{1} & =\frac{t_{\mathrm{sim}}+t_{\mathrm{su}} S\left(1-\beta^{N-1}\right)}{S-\beta^{N-1}(S-1)}, \\
t_{i} & =\frac{(S-1) /(S+1) t_{\mathrm{sim}}-\beta t_{\mathrm{su}}}{S-(S-1) \beta^{N-1}} \times \beta^{i-2}, \\
& i \geqslant 2,
\end{aligned}
$$

where $\beta=S /(S+1)$.

We simulated a small and a large gate level model of a pipelined multiplier as well as two models of the ISCAS89 benchmark suite (s1196 and s1238) on our system. The models include extra code to check setup and hold conditions of the flipflops. The small multiplier model consists of $\approx 5500$ elements (gates + flipflops), the large model consists of $\approx 10,000$ elements, s1196 and s1238 are each made of $\approx 500$ elements. Table 2 shows the $S$ values of measured for the different models.

As the computation nodes do not communicate during parallel simulation we emulated parallel simulation by executing the simulation programs (cycle based and timing) of each node subsequently on a single machine. The parallel simulation runtime was determined as the maximum runtime among all this simulations. The speedup is the ratio between the runtime of a single sequential timing simulation and the emulated parallel simulation time.

Fig. 6 shows the simulation results measured on a PC running Linux (133 MHz Pentium). The startup time was set to two cycles, the time slices assigned to the nodes were calculated as described above.

Fig. 7 presents the standard deviation of the simulation runtimes measured for the different

Table 2

$S$ values of the example models

\begin{tabular}{lllll}
\hline & $\begin{array}{l}\text { Small } \\
\text { mult. }\end{array}$ & $\begin{array}{l}\text { Large } \\
\text { mult. }\end{array}$ & s1196 & s1238 \\
$S$ & 69.48 & 77.67 & 16.59 & 14.77 \\
\hline
\end{tabular}

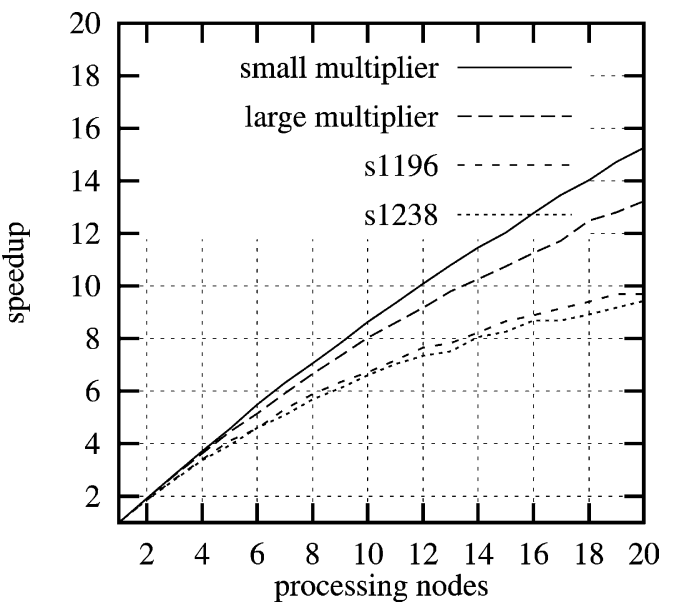

Fig. 6. Measured speedups for various models.

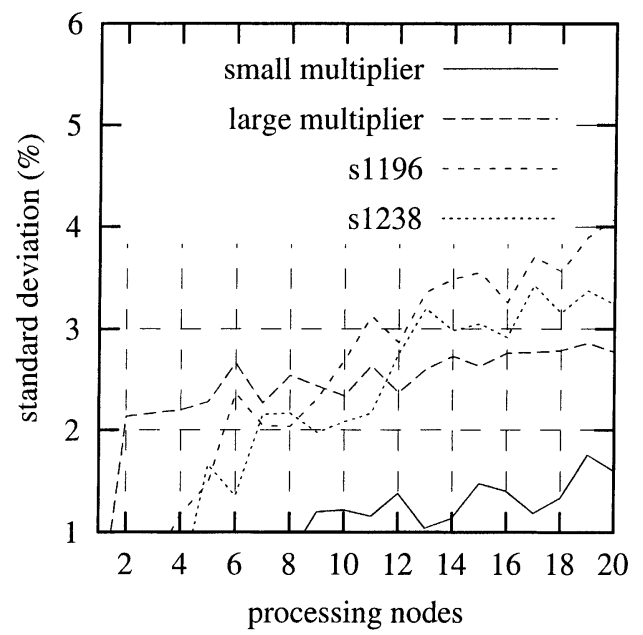

Fig. 7. Standard deviation of the simulation times.

computation nodes. The results has been related to the average runtime of all nodes. Figs. 8 and 9 compare the theoretical and the measured speedup results for the multiplier and the ISCAS models.

\section{Discussion}

As shown in Fig. 6 even for the small models an excellent speedup was achieved. We expect similar or better results with larger models. Further, as the 


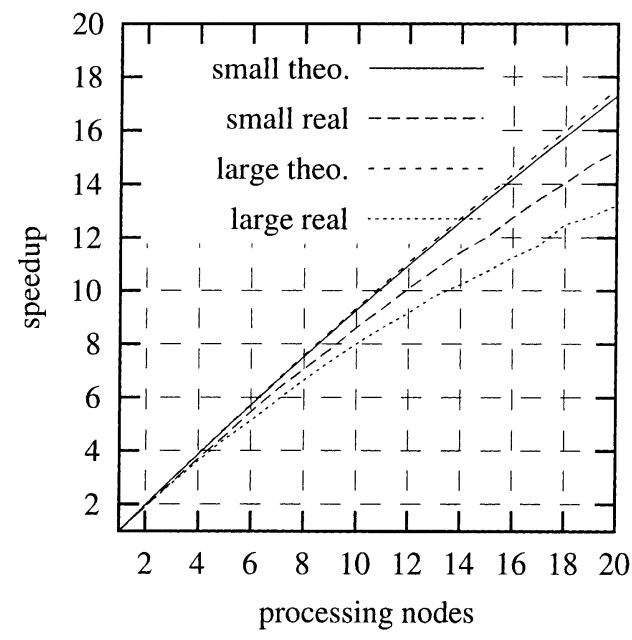

Fig. 8. Theoretical versus real speedup for the multiplier models.

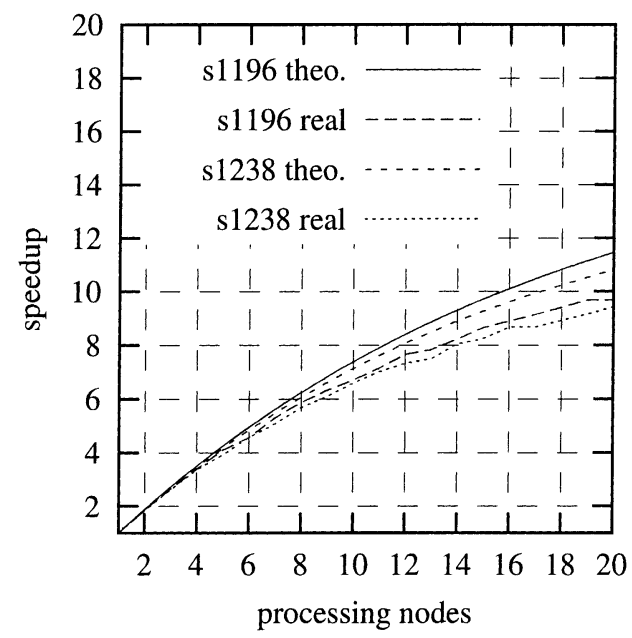

Fig. 9. Theoretical versus real speedup for the ISCAS models.

state information is currently transfered from cycle simulator to the timing simulator via file $\mathrm{I} / \mathrm{O}$ there is still room for further improvement.

A significant advantage of our approach is that the cycle based simulator as well as the timing simulator are actually based on common serial simulation algorithms. Only some minor modifications are necessary to store and read state information. Hence, any techniques to speed up cycle based as well as timing simulation can be incorporated into our approach.
Compared to a serial simulation run there is some overhead which has to be taken into account when analyzing the speedup of our approach. First, state information has to be produced by the cycle simulators and transfered to the timing simulators. Further, while timing simulation runtime can be reduced by using additional processing nodes, the elaboration runtime cannot be decreased. Usually, timing simulation is divided into two phases. During the initial phase the structure of the model is elaborated, i.e., the elements are constructed in the memory of the simulation computer from the HDL description. In the next phase the elaborated model is simulated. While the time spent in simulation phase can be reduced by distributing the workload on several computation nodes, the entire initial phase is executed by each timing simulator thus further limiting the speedup. However, usually the initial phase consumes significantly less computation time than the actual simulation phase for most models.

Differences between theoretical and measured speedups are due to two main reasons. First, for the theoretical results the elaboration time was not taken into account. While for small $N$ elaboration usually makes up only a small part of the total simulation time it becomes more and more significant with growing $N$. Further, the runtime spent in the elaboration phase usually increases with the circuit size. Hence, the most distinct differences between theoretical and experimental results has been measured for the large multiplier followed by the small multiplier (see Fig. 8) and the ISCAS models (see Fig. 9).

Another source for differences between theoretical and measured results is the data dependency of timing simulation. Usually, the workload for timing simulation is not strictly proportional to the simulation time span but also depends on the input data presented to the circuit. Hence, as shown in Fig. 7 the runtime of the timing simulations varies. However, even the worst sample does have a standard deviation of less than $4 \%$. Obviously by using Eq. (6) to calculate the time slices a good uniform utilization could be achieved for all computation nodes. 
A major disadvantage of our approach is that only synchronous circuits can be simulated. Further, each computation node must have enough memory to store the entire simulation model. Finally, it may be required to modify the testbench in order to be able to use our technique. In the worst case a cycle based compliant testbench must be written for cycle simulation and another testbench for timing simulation. However, in many cases, e.g., when only input stimuli are generated by the testbench and the outputs are stored or compared against the expected responses, the testbench can be reused for both simulation types. Further, our approach is well suited for NOWs as no communication takes place during simulation. Only the simulation executable and any output data generated by the models during simulation must be transferred to or from the computation nodes. However, this is usually done before or after the actual simulation run. Hence, the speedup of our technique is not dependent on any efficient communication network.

Our method make NOWs suitable for parallel timing simulation. Hence, even huge simulation workloads (e.g., to backup static timing analyzer results or generate information for power consumption calculation) can be handled efficiently with NOWs. Moreover, as no communication is required during parallel simulation the network bandwidth available for any other applications running on other nodes of the NOWs is preserved. Hence, no dedicated simulation "farms" are needed. Further, as the simulation speedup is not dependent on communication bandwidth or latency the computation power of idle machines in a (heterogenous) network can be exploited efficiently.

\section{Related work}

Common parallel simulation techniques require a significant amount of communication in order to synchronize the simulators running on the different computation nodes $[1-3,7]$. This includes sending events that are generated by a node and are targeting an element on another node. Further, additional messages are transmitted to publish the state of the various nodes. This information is needed in order to preserve the correctness of the simulation results. Conservative approaches use this information to ensure that all events are processed in time stamp order [7,11]. Optimistic approaches are using it to remove old state backups from memory and to determine the current virtual simulation time [7].

Our approach is conservative as it preserves the correctness of the simulation. Contrary to other techniques our method does not rely on information received from other computation nodes to synchronize simulation. No events and no synchronization information are exchanged between computation nodes. This makes our technique especially well suited for parallel simulation on NOWs. However, for our approach the maximum speedup is limited by $S$. Other approaches may achieve higher speedup values, but they usually rely on expensive high speed networks $[4,10]$.

While common parallel simulation algorithms produce exactly the same results as a corresponding serial one, our algorithm behaves differently for faulty models. If a setup violation occurs during simulation within a specific time slice a wrong value is sampled by the corresponding flipflop. Usually, this will make all subsequent simulation useless as wrong data are now processed by the circuit. However, in our approach cycle simulation is used to determine the starting state for all time slices. As each time slice starts with valid data (as intended by the designer) an error occurring in a specific time slice does not affect simulation of the other slices. Hence, the remaining slices may still produce useful information which is a significant advantage in most cases. Nevertheless, simulating timing correct models (i.e., models which do not include any timing errors) with our approach gives exactly the same results as generated by a serial discrete event simulation algorithm.

Contrary to our simulation algorithm other common PDES techniques often require a special parallel simulation kernel. This makes it difficult or even impossible to incorporate some of the optimization techniques developed for serial simulation. However, our algorithm is restricted 
to synchronous circuits while other common approaches can be usually applied to all circuit types. But, as the majority of modern designs are synchronous this is not a significant drawback.

\section{Conclusion}

We have developed a new parallel simulation algorithm for timing verification of synchronous digital circuits. Our approach exploits the performance gap between cycle based simulators and timing simulator techniques. In order to run several timing simulators in parallel we use cycle based simulation to produce the synchronization information. Thus, each computation node of the parallel computer (timing) simulates the entire circuit for a different time slice. In order to achieve maximum speedup we developed a framework to determine the time slices to be associated with each individual node.

A major advantage of our approach is that no communication between the computation nodes is required during simulation. This makes this technique suitable for parallel simulation on NOWs. We implemented the approach which shows excellent speedup results compared to a serial timing simulator.

\section{References}

[1] R. Ayani, H. Rajaei, Parallel simulation based on conservative time windows: a performance study, Concurrency: Practice and Experience 6 (2) (1994) 119-142.

[2] M.L. Bailey, J.V. Briner, R.D. Chamberlain, Parallel logic simulation of VLSI systems, ACM Computing Surveys 26 (3) (1994) 255-294.

[3] K.M. Chandy, J. Misra, Asynchronous distributed simulation via a sequence of parallel computations, Communications of the ACM 24 (11) (1981) 198-206.

[4] Y. Chen, V. Jha, R. Bagrodia, A multidimensional study on the feasibility of parallel switch-level circuit simulation, in: Proceedings of the 11th Workshop on Parallel and Distributed Simulation (PADS'97), Lockenhaus, Austria, 1997.

[5] Y. Chen, R. Bagrodia, Shared memory implementation of a parallel switch-level circuit simulator, in: Proceedings of the 12th Workshop on Parallel and Distributed Simulation (PADS'98), Banff, Alberta, Canada, 1998.
[6] R.S. French, M.S. Lam, J.R. Levitt, Kunle Olukotun: a general method for compiling event-driven simulations, in: Proceedings of the 32nd Design Automation Conference (DAC 1995), San Francisco, CA, USA, June 1995, pp.151156.

[7] R.M. Fujimoto, Parallel discrete event simulation, Communication of the ACM 33 (10) (1990) 30-53.

[8] D.R. Jefferson, Virtual time, ACM Transaction on Programming Languages and Systems 7 (3) (1985) $404-425$.

[9] N. Manjikian, W.M. Loucks, High performance parallel logic, simulation on a network of workstations, in: Proceedings of the 7th Workshop on Parallel and Distributed Simulation (PADS'93), San Diego CA, 1993, pp. 7684.

[10] E. Naroska, U. Schwiegelshohn, Conservative parallel simulation of a large number of processes, Simulation 172 (3) (1999) 150-162.

[11] J. Misra, Distributed discrete-event simulation, Computing Surveys 18 (1) (1986) 39-65.

[12] Quickturn Design Systems, Inc., Speed-Sim Brochure, [Online] Available http://www.quickturn.com/.

[13] R. Radhakrishnan, N. Abu-Ghazaleh, M. Chetlur, P.A. Wisley, On-line configuration of a time warp parallel discrete event simulator, in: Proceedings of the International Conference on Parallel Processing (ICPP'98), Minneapolis, August 1998.

[14] L. Soulé, A. Gupta, An evaluation of the Chandy-MisraBryant algorithm for digital logic simulation, ACM Transaction on Modeling and Computer Simulation 1 (4) (1991) 308-347.

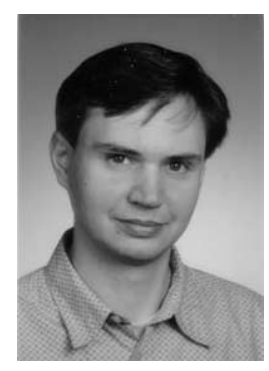

Edwin Naroska has been an Assistant Professor at the Computer Engineering Institute of University Dortmund since 1998. He received a Diploma in Electrical Engineering in 1990 and a Doctorate Degree in 1997, both from the University of Dortmund. His main research interests include VLSI design, VLSI verification and computer architecture.

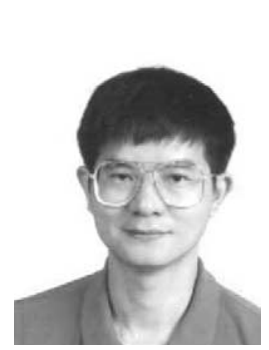

Feipei Lai received a B.S.E.E. degree from National Taiwan University in 1980, and M.S. and Ph.D. degrees in Computer Science from the University of Illinois at Urbana-Champaign in 1984 and 1987, respectively. He is a professor in the Department of Electrical Engineering and in the Department of Computer Science and Information Engineering at National Taiwan University. His current research interests are high performance microprocessor chip design, computer architecture, optimizing compiler, VLSI design. 


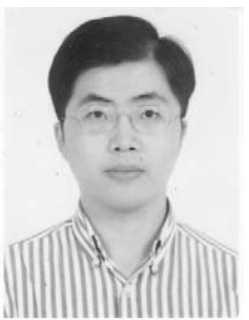

Rung-Ji Shang received the B.S. degree from National Chiao Tung University in 1990, the M.S. and Ph.D. degrees from National Taiwan University in 1992 and 2000, respectively. He is presently a product developer in Synology Incorporated. His research interests include parallel processing, system programming and computer architecture.

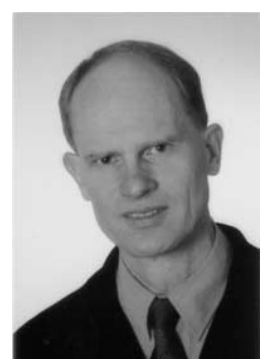

Uwe Schwiegelshohn is a Professor at the Computer Engineering Institute of the University Dortmund since 1994. He received a Diploma and a Doctor degree in Electrical Engineering from the Technical University Munich in 1984 and 1988, respectively. From 1988 to 1994 he was with the IBM T.J. Watson Research Center in Yorktown Heights. His main research interests include scheduling problems, parallel computing, management of computer systems and embedded systems. 